\title{
T2-FLAIR Mismatch Sign and Response to Radiotherapy in Diffuse Intrinsic Pontine Glioma
}

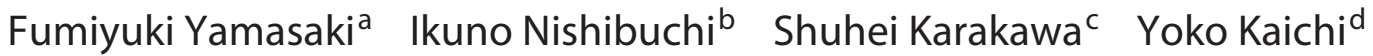

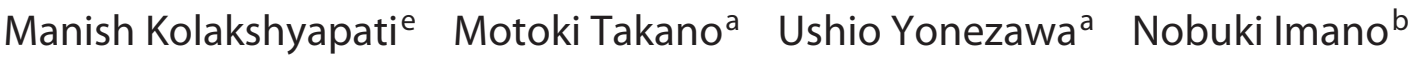 \\ Akira Taguchi $^{a}$ Maiko Shimomura ${ }^{c}$ Maki Taniguchic Shumpei Onishia \\ Satoshi Okadac Kazuo Awai $^{d}$ Kazuhiko Sugiyama ${ }^{f}$ Yasushi Nagata ${ }^{b}$ \\ ${ }^{a}$ Department of Neurosurgery, Hiroshima University Hospital, Hiroshima, Japan; bepartment of Radiation \\ Oncology, Hiroshima University Hospital, Hiroshima, Japan; 'Department of Pediatrics, Hiroshima University \\ Hospital, Hiroshima, Japan; 'Department of Diagnostic Radiology, Hiroshima University Hospital, Hiroshima, Japan; \\ eDepartment of Neurosurgery, B\&B Hospital, Gwarko, Lalitpur, Nepal; f Department of Clinical Oncology and \\ Neuro-Oncology Program, Hiroshima University Hospital, Hiroshima, Japan
}

\section{Keywords}

T2-FLAIR mismatch - Diffuse intrinsic pontine glioma •

Diffuse midline glioma · Imaging marker · Pediatric brain tumors

\section{Abstract \\ Purpose: The T2-fluid-attenuated inversion recovery (FLAIR) mismatch sign was previously reported as a diagnostic indi- cator of diffuse astrocytoma, isocitrate dehydrogenase-mu- tant, and $1 p / 19 q$ noncodeletion. Subsequently, it was re- ported that the same findings were observed in diffuse in- trinsic pontine glioma (DIPG). We investigated the clinical significance of T2-FLAIR mismatch sign in DIPG. Methods: Twenty-one patients with DIPG (Male: Female $=12: 9$ ) were treated at our institute between 2004 and 2019. All patients were treated with local radiotherapy of $54 \mathrm{~Gy} / 30$ fractions. The positive T2-FLAIR mismatch sign was defined if it fulfilled the following criteria: (1) T2-FLAIR mismatch volume was $>50 \%$ of T2 high volume at nonenhanced area, (2) the FLAIR low lesion is not associated with gadolinium enhancement}

(inside of enhancement or just outside of enhancement defined as edema), and (3) signal-intensity of FLAIR lowest lesion at tumor is lower than the normal cerebellar cortex. $R \boldsymbol{e}$ sults: In our patient series, T2-FLAIR mismatch sign was found in 5 out of 21 patients. Objective response rate of radiotherapy was $100 \%$ in patients positive for T2-FLAIR mismatch, while it was $25.0 \%$ in patients negative for T2-FLAIR mismatch, and this difference was statistically significant ( $p<0.01$, Fisher's exact test). In patients under the age of 18-years, T2-FLAIR mismatch positive had a slightly better prognosis ( $p<0.05$, Wilcoxon test). Conclusion: T2-FLAIR mismatch sign in DIPG may be an indicator for better response to radiotherapy and a better prognostic factor.

(c) 2021 S. Karger AG, Basel

\section{Introduction}

Diffuse intrinsic pontine glioma (DIPG) accounts for $10-15 \%$ of childhood brain tumors and up to $80 \%$ of pediatric brain-stem tumors [1]. Despite treatment efforts 
using chemotherapy and radiotherapy, the median overall survival (OS) remains around 1 year from diagnosis and remains as one of the most difficult tumors to treat successfully. The management strategies other than local irradiation are ineffective, with the benefit of irradiation being transient at best [2]. The anatomic location and infiltrative nature of DIPG severely limit any opportunity for fruitful surgical resection to impact prognosis, and no effective chemotherapy has been reported to improve progression or OS $[3,4]$. A previous study reported the long-term survivors of DIPG were aged $<3$ or $>10$ years and had long symptom duration, while the short-term survivors presented with symptoms of cranial nerve palsy and had ring enhancement, necrosis, and extrapontine extension on imaging [5]. However, these data do not help in decision-making for the initial treatment for DIPG.

The "T2-FLAIR (fluid-attenuated inversion recovery) mismatch sign" was recently reported as an imaging biomarker for diagnosis of diffuse glioma, isocitrate dehydrogenase (IDH)-mutant, and $1 \mathrm{p} / 19 \mathrm{q}$ noncodeleted. Several studies demonstrated that the specificity of T2FLAIR mismatch sign is high for diagnosis of diffuse astrocytoma with IDH-mutant genomic alteration [6]. However, the previous studies for T2-FLAIR mismatch were confirmed only among lower grade glioma, in other words, "diffuse astrocytic and oligodendroglial tumors" [7-10]. Subsequent studies demonstrated that this sign could be observed in other gliomas including diffuse midline glioma, H3 K27M mutant of the thalamus [11]. However, there has been no report about T2-FLAIR mismatch sign in DIPG. In this study, we focused on the clinical implication of T2-FLAIR mismatch sign observed in a subset of DIPG. We found that T2-FLAIR mismatch sign in DIPG is an indicator for better response to radiotherapy.

\section{Materials and Methods}

\section{Patients Selection}

Our institutional review board approved this retrospective study (IRB No. 2922/E-1650). To protect patient privacy, we removed all identifiers from our records upon completion of the analyses. Between 2004 and 2019, 21 patients with DIPG (12 male and 9 females) were treated at our institute. We excluded the patients with diffuse midline glioma, H3 K27M-mutant at thalamus, because age, clinical characteristics, surgical intervention and prognosis of diffuse midline glioma at thalamus are much different from DIPG, even though the genetic pathway reportedly resembles the histone 3 mutation.

The patients' age of DIPG ranged from 3 to 36 (median 6) years. The number of patients $<18$ years was 18 (age range $3-17$, median 6 years). All patients underwent MRI study which included nonenhanced T1-weighted imaging (T1WI), T2-weighted imaging (T2WI), FLAIR, diffusion-weighted imaging, and gadolinium-enhanced T1WI with slice thickness $7 \mathrm{~mm}$ or less, at least every 2 months until recurrence. Recurrence was defined as imaging progression with or without symptomatic progression. All patients were treated with local radiotherapy of $54 \mathrm{~Gy} / 30$ fractions after the imaging diagnosis of DIPG. The details about day of onset, confirmation of diagnosis (MR examination date for diagnosis), and death were obtained from medical records. The progression-free survival and the OS were calculated by using the date of diagnosis.

\section{MR Acquisition and Evaluation}

MR scans were acquired at 1.5 or $3.0 \mathrm{~T}$ scanners and assessed by 2 independent authors (S.O. and F.Y.). Investigators evaluated the T1WI, T2WI, FLAIR, and post-contrast T1WI sequencing of each patient. In this study, the positive T2-FLAIR mismatch sign was defined if it fulfilled the following criteria: (1) T2-FLAIR mismatch volume was $>50 \%$ of T2 high volume at nonenhanced area, (2) the lesion of FLAIR low is not associated with gadolinium enhancement (inside of enhancement or just outside of enhancement defined as edema), and (3) signal-intensity of FLAIR lowest lesion at tumor is lower than the normal cerebellar cortex and not the white matter. The cutoff point of $50 \%$ about T2-FLAIR mismatch volume was defined based on the previous report [8]. On visual inspection, the FLAIR low is highly affected by the surrounding tissue intensity, and therefore, we strictly calculated the intensity of the tumor lesions. Both investigators independently assessed (i) presence or absence of the T2-FLAIR mismatch sign and (ii) presence or absence of gadolinium contrast enhancement. We also defined the areas of visually lower intensity of tumor and intensity of normal-appearing cerebellar cortex on FLAIR image by consensus and calculated the ratio.

Single-voxel proton MR spectroscopy (1H-MRS) was performed as described previously [12]. In brief, 1H-MRS was performed with the point-resolved spectroscopic method (PRESS; repetition time, $2,000 \mathrm{~ms}[3.0 \mathrm{~T}] ; \mathrm{TE}=144$ and $30 \mathrm{~ms}$; data points, 2,048; signals acquired, 64 [3.0 T]; double-spin-echo sequence) on a 3.0 T superconducting system, versions 12 and 15 (Signa Excite HD 3.0 T or Ingenia CX 3.0 T R5.1.2); a circularly polarized head coil or Ds Head 32ch coil was used as described previously. Singlevoxel spectroscopic examinations were guided using enhanced T1weighted images. Under 3D control, the rectangular $1 \mathrm{H}-\mathrm{MRS}$ voxel was placed on the gadolinium-enhanced tumor area if present. The relative SIs of choline, $\mathrm{Cr}$, and $\mathrm{N}$-acetyl aspartate were obtained by numeric integration of fitted signals.

The effect of radiotherapy on tumor was determined according to Response Assessment in Neuro-Oncology criteria as follows: complete response (complete disappearance), partial response (reduction by $\geq 50 \%$ ), stable disease (size change $<25 \%$ ), and progressive disease (increase by $\geq 25 \%$ ).

\section{Statistical Analysis}

Statistical analyses were performed with SPSS pro ver. 21.0. The relationship between response to irradiation and T2-FLAIR mismatch sign was evaluated by Fisher's exact test. The Kaplan-Meier analysis was used, and statistical significance was assessed using a log-rank test and Gehan-Breslow-Wilcoxon test; $p<0.05$ was considered statistically significant. 


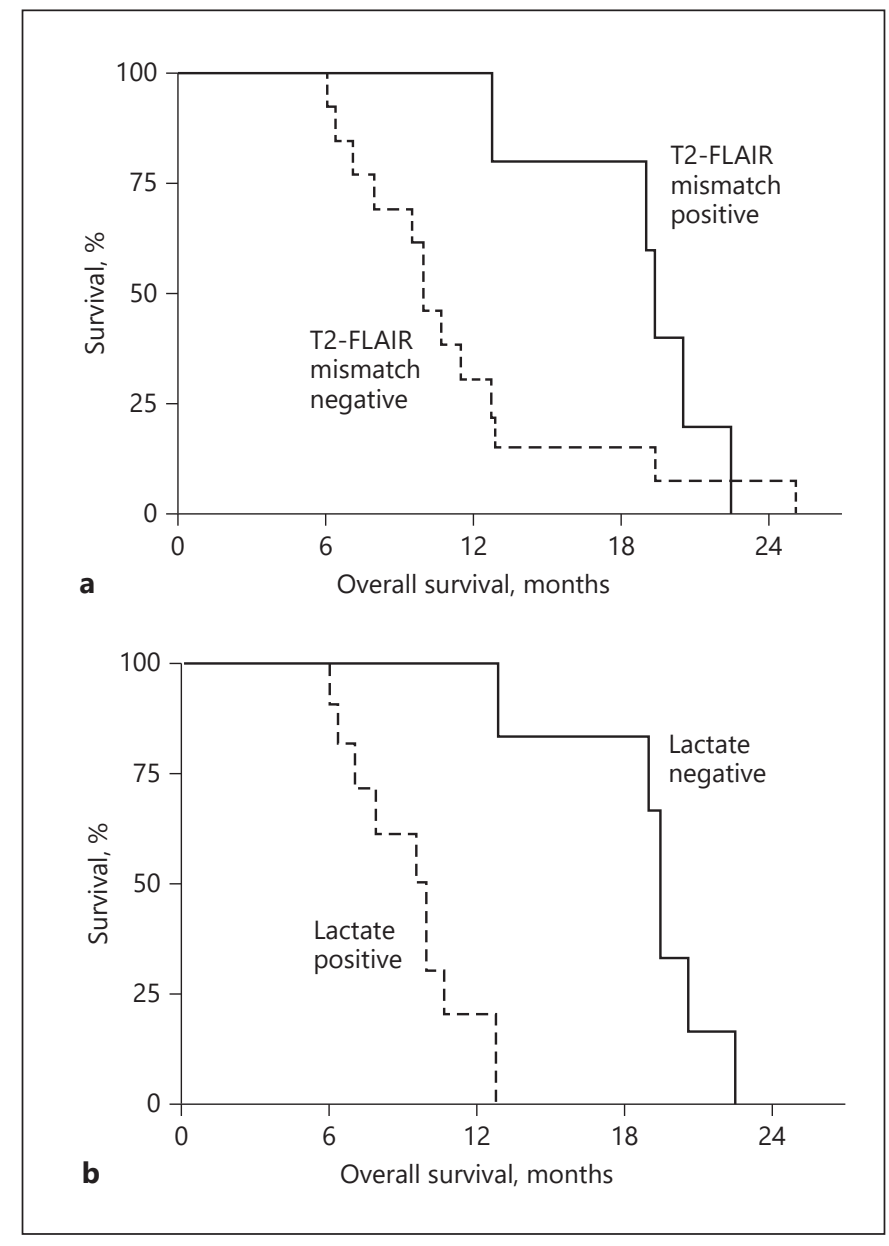

Fig. 1. Kaplan-Meier survival curve of DIPG patients treated with local radiotherapy. a Under age of 18 years, T2-FLAIR mismatch positive patients showed better survival than T2-FLAIR mismatch negative patients ( $p=0.0352$, Gehan-Breslow-Wilcoxon test). b Lactate detection was significantly associated with poor prognosis in patients under 18 years $(p=0.0002$ at log-rank test and $p=$ 0.0012 at Gehan-Breslow-Wilcoxon test. DIPG, diffuse intrinsic pontine glioma; FLAIR, fluid-attenuated inversion recovery.

\section{Results}

Clinical course and imaging characteristics of patients with T2-FLAIR match and mismatch are summarized in Table 1. Five patients had the characteristics T2-FLAIR mismatch and the ratio was $23.8 \%$ (5/21) among all patients, and $27.8 \%(5 / 18)$ among patients under the age of 18 years. In pediatric patients, the age at onset of T2FLAIR mismatch positive patients (mean 5.8 and median 5 ) was younger than those without T2-FLAIR mismatch (mean 8 and median 6). No adolescent and young adult (AYA) patients showed the characteristics of T2-FLAIR mismatch.

T2-FLAIR Mismatch Sign in DIPG

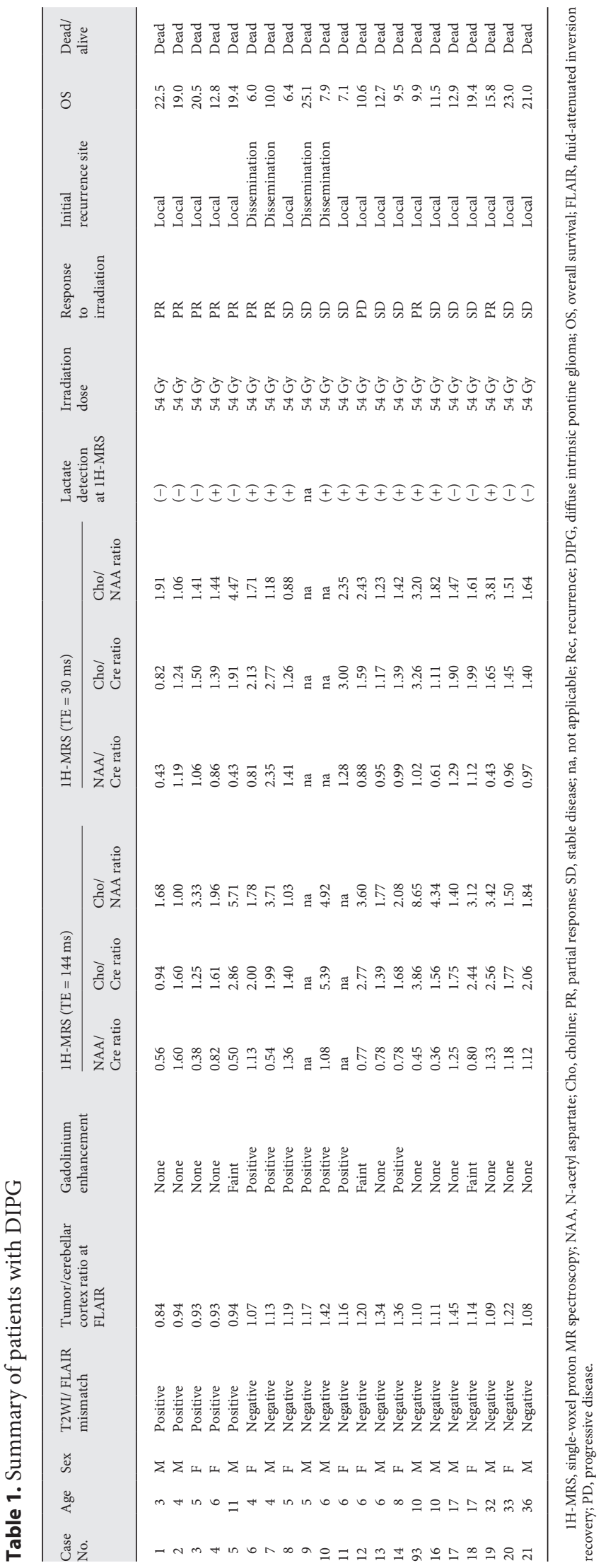

Pediatr Neurosurg 2021;56:1-9 


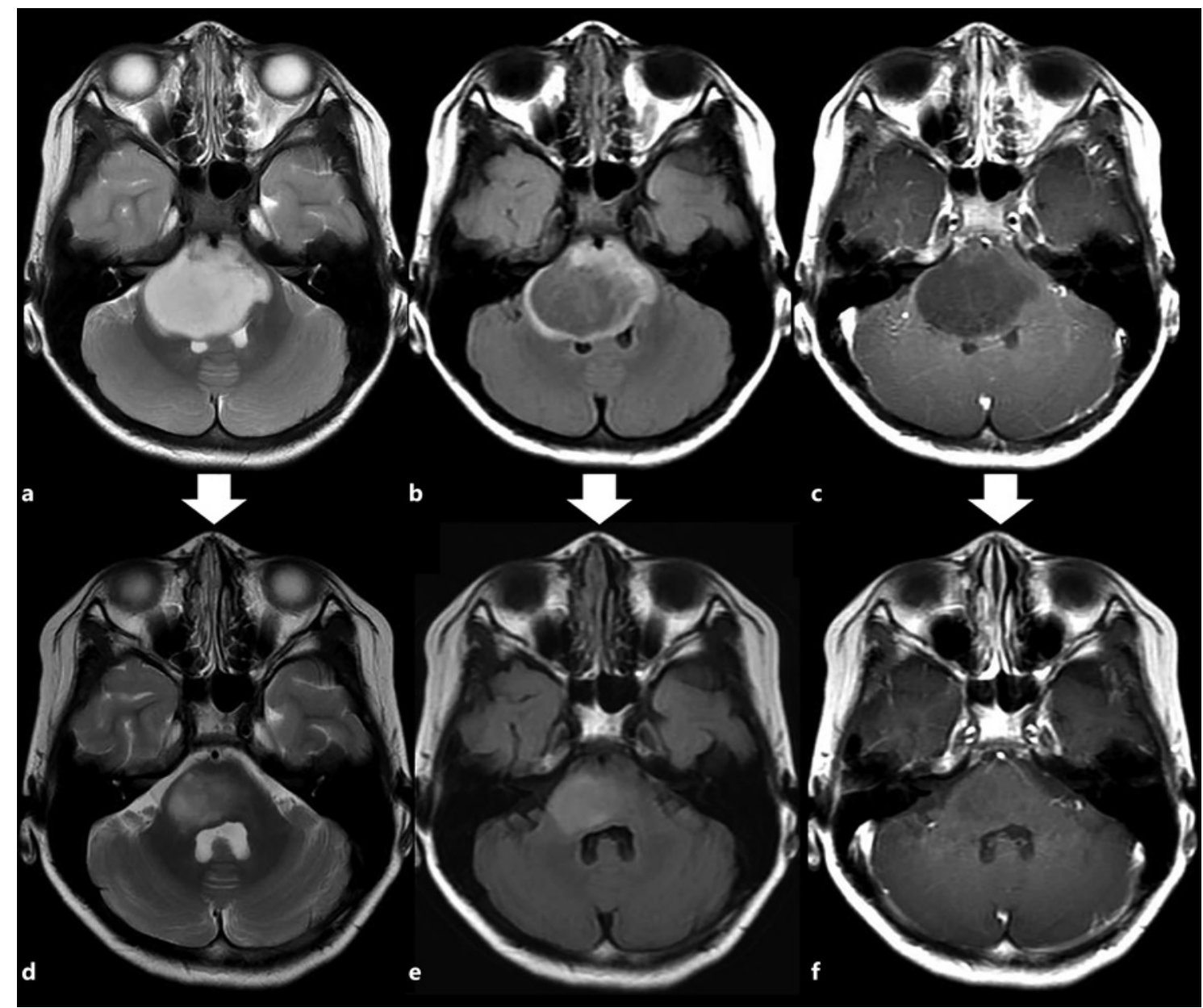

Fig. 2. Representative case of 5-year-old girl with T2-FLAIR mismatch sign in DIPG (case No. 3). Pre- (a-c) and post-RT (d-f) images. Axial T2WI (a, d), FLAIR images $(\mathbf{b}, \mathbf{e})$, and gadoliniumenhanced image $(\mathbf{c}, \mathbf{f})$ of a patient with DIPG. a T2WI demonstrates homogeneous high-intensity tumor throughout the lesion. b FLAIR image demonstrates lower intensity at majority of tumor compared to cerebellar cortex. c Gadolinium enhanced T1WI demonstrates homogenous low-intensity tumor with no enhancement. d-f Tumor showed PR after irradiation. DIPG, diffuse intrinsic pontine glioma; FLAIR, fluid-attenuated inversion recovery; PR, partial response.
All patients underwent local radiotherapy of $54 \mathrm{~Gy} / 30$ fractions. Objective response rate of radiotherapy was $100 \%$ in patients positive for T2-FLAIR mismatch, while it was $25.0 \%$ in patients negative for T2-FLAIR mismatch, and this difference was statistically significant $(p=0.0062$, Fisher's exact test). In patients under the age of 18 years, the statistical difference of objective response among T2-FLAIR mismatch positive patients was also statistically significant $(p=0.0065$, Fisher's exact test). Other imaging characteristics including the cyst formation, gadolinium enhancement, and parameters of $1 \mathrm{H}$-MRS were not associated with response to irradiation.
In patients under the age of 18 years, T2-FLAIR mismatch positive had a slightly better prognosis $(p=$ 0.0352, Gehan-Breslow-Wilcoxon test, Fig. 1a), while, in the analysis of all patients, T2-FLAIR mismatch sign did not reach statistical significance as the AYA patients showed the tendency of better prognosis. All 5 T2-FLAIR mismatch positive tumors recurred at the original tumor site. We also evaluated the prognostic value of other parameters. Lactate detection was significantly associated with poor prognosis in both pediatric and AYA patients $(p<0.0001$ at log-rank test and $p=0.0002$ at Gehan-Breslow-Wilcoxon test) and under age of 18 -year patients $(p=0.0002$ at log-rank test 


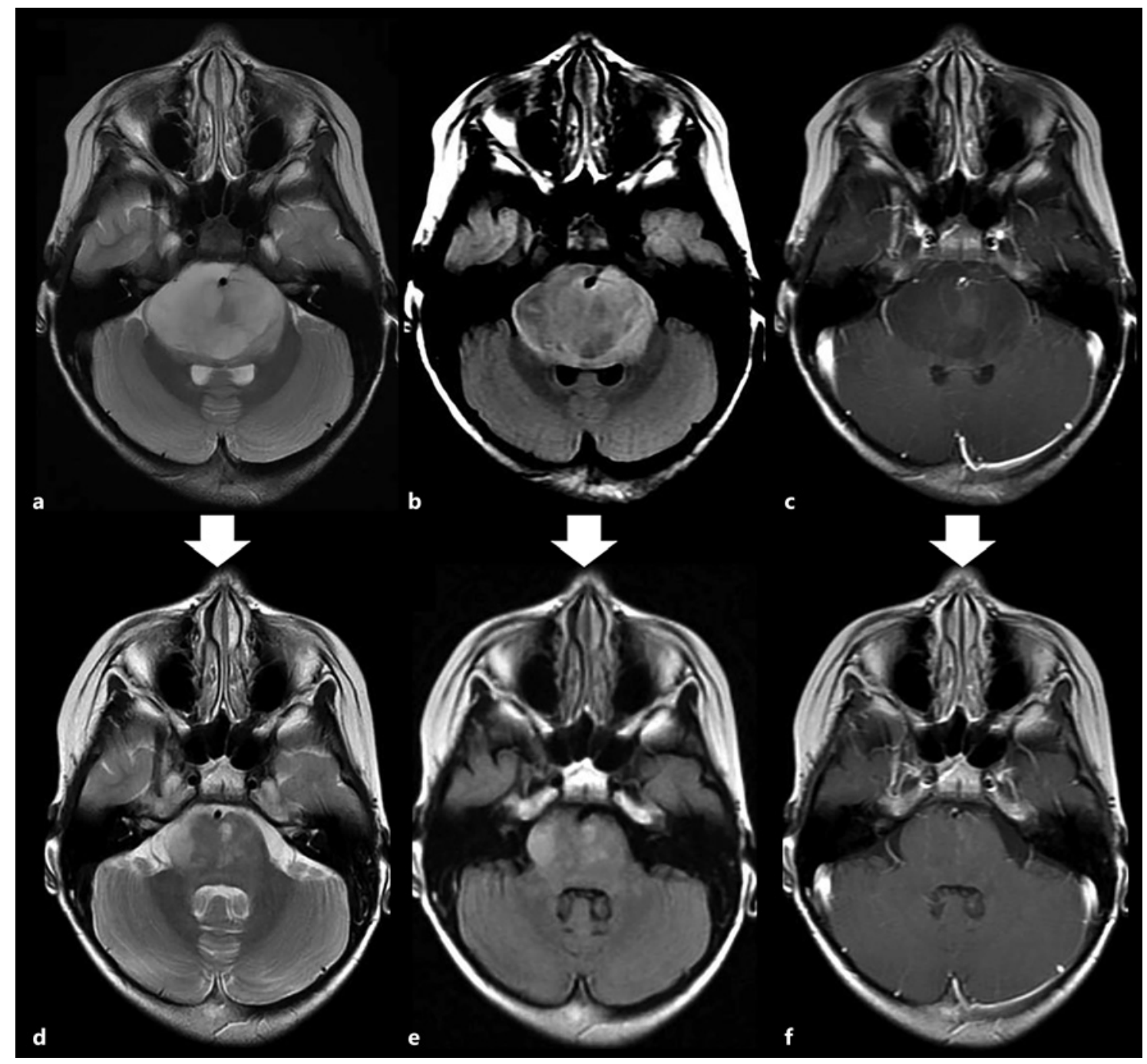

Fig. 3. Representative case of 6-year-old girl with T2-FLAIR mismatch sign in DIPG (case No. 4). Pre- (a-c) and post-RT (d-f) images. Axial T2WI $(\mathbf{a}, \mathbf{d})$, FLAIR images $(\mathbf{b}, \mathbf{e})$, and gadolinium-enhanced image $(\mathbf{c}, \mathbf{f})$ of a patient with DIPG. a T2WI demonstrates homogeneous high-intensity tumor throughout the lesion. b FLAIR image demon- strates lower intensity at majority of tumor compared to cerebellar cortex. c Gadolinium enhanced T1WI demonstrates homogenous low-intensity tumor with partial faint enhancement. d-f Tumor showed PR after irradiation. DIPG, diffuse intrinsic pontine glioma; FLAIR, fluid-attenuated inversion recovery; PR, partial response. and $p=0.0012$ at Gehan-Breslow-Wilcoxon test, Fig. 1b).

We finally evaluated the relationship between T2FLAIR mismatch sign and $1 \mathrm{H}-\mathrm{MRS}$ parameters. The T2FLAIR mismatch tumor showed the tendency of lower choline/Cr ratio. However, we could not find any statistical difference of $1 \mathrm{H}-\mathrm{MRS}$ parameters between T2-FLAIR mismatch positive and negative patients. Representative cases with T2-FLAIR mismatch sign are shown in Figures 2 and 3 . And representative cases without T2-FLAIR mismatch sign are shown in Figures 4 and 5.

T2-FLAIR Mismatch Sign in DIPG
Pediatr Neurosurg 2021;56:1-9

DOI: $10.1159 / 000513360$

\section{Discussion}

The typical imaging feature of DIPG is high intensity on T2WI and low to iso-intensity on T1WI, with nondelineated tumor margins and engulfment of the basilar artery [13]. On FLAIR images, DIPGs usually appear homogeneously hyperintense [14]. However, there have been no reports that focused on FLAIR images. In this study, our results indicate that T2-FLAIR mismatch sign in DIPG could be a possible indicator for better response to radiotherapy. To our knowledge, this is the first report 


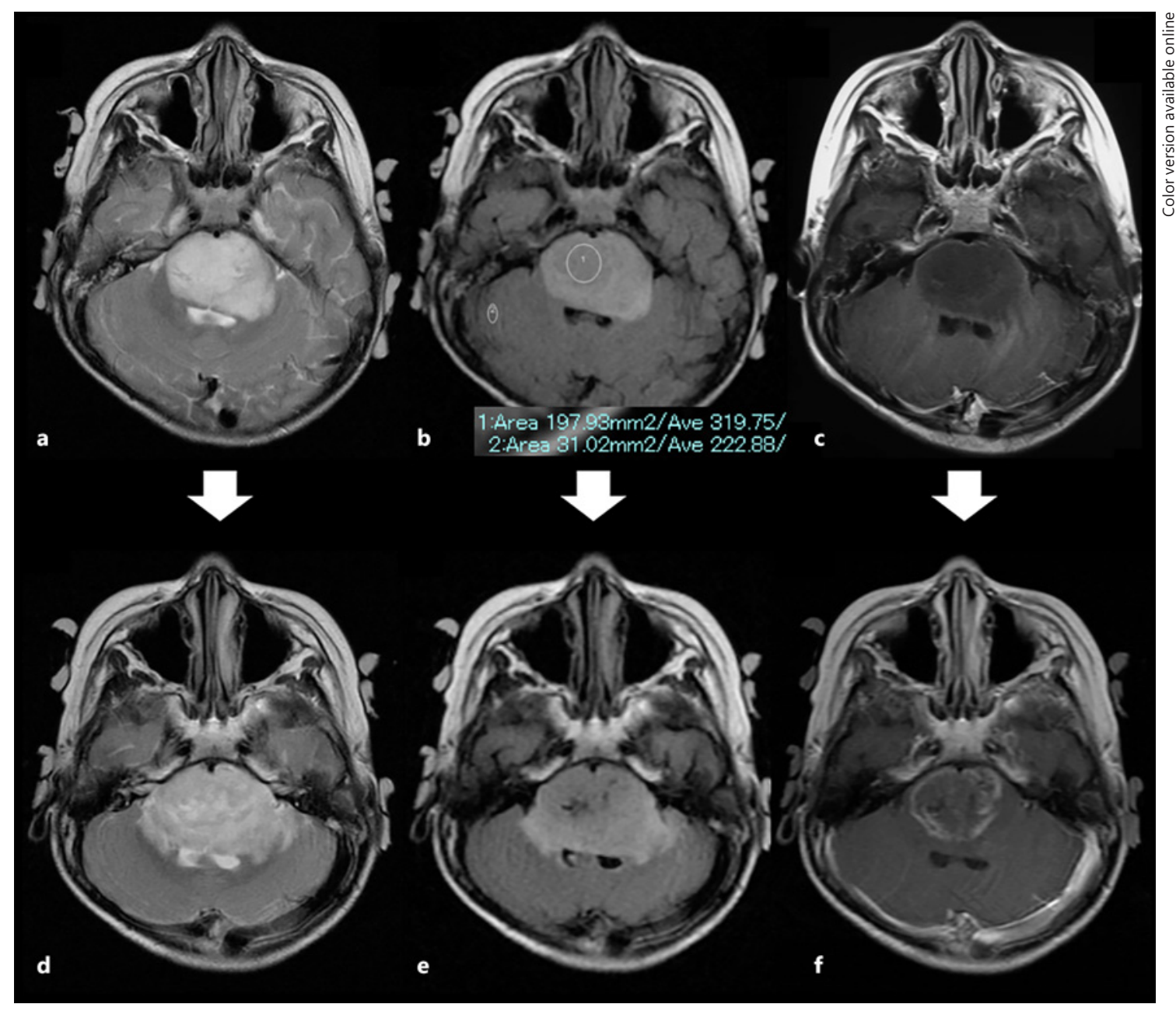

Fig. 4. Representative case of 6-year-old boy without T2-FLAIR mismatch sign in DIPG (case No. 13). Pre- (a-c) and post-RT (df) images. Axial T2WI (a, d), FLAIR images $(\mathbf{b}, \mathbf{e})$, and gadolinium-enhanced image $(\mathbf{c}, \mathbf{f})$ of a patient with DIPG. a T2WI of preradiotherapy demonstrates homogeneous high-intensity tumor throughout the lesion. b FLAIR image of pre-radiotherapy demonstrates iso- to high intensity at tumor and the signal intensity is higher compared to cerebellar cortex. The ratio of visually lower intensity inside the tumor and visually higher intensity at cerebellar cortex was 1.43 and confirmed that FLAIR intensity of tumor is higher than cerebellar cortex. c Gadolinium enhanced T1WI demonstrates homogenous low-intensity tumor with no enhancement. d-f Tumor showed no response after irradiation. DIPG, diffuse intrinsic pontine glioma; FLAIR, fluid-attenuated inversion recovery. which showed that a subgroup of DIPG potentially has the merit with increased dose intensity of radiotherapy. Our data would be useful for the development of treatment strategy with radiotherapy for DIPG.

Radiotherapy is the only treatment that has shown benefit in the treatment of DIPG both for newly diagnosed and recurrence/refractory cases although it is essentially considered as palliative therapy [15]. Many clinical trials have been conducted over several decades for DIPG [1]. It included hyper-fractionated radiotherapy, hypo-fractionated radiotherapy, with or without, concur- rent, concomitant chemotherapy, or chemotherapy immediately after radiotherapy. However, there are no reports that showed improved results in patients with DIPG and outcomes remained largely unchanged. The benefit of palliative re-irradiation for recurrent progressive DIPG was reported in both retrospective and recurrent settings [15-21]. However, re-irradiation further requires assessing the optimal dose, fractionation, the interval between upfront RT and re-irradiation, and the persistent period of symptom improvement. Moreover, the failure of the clinical trials with upfront irradiation dose-dense setting 


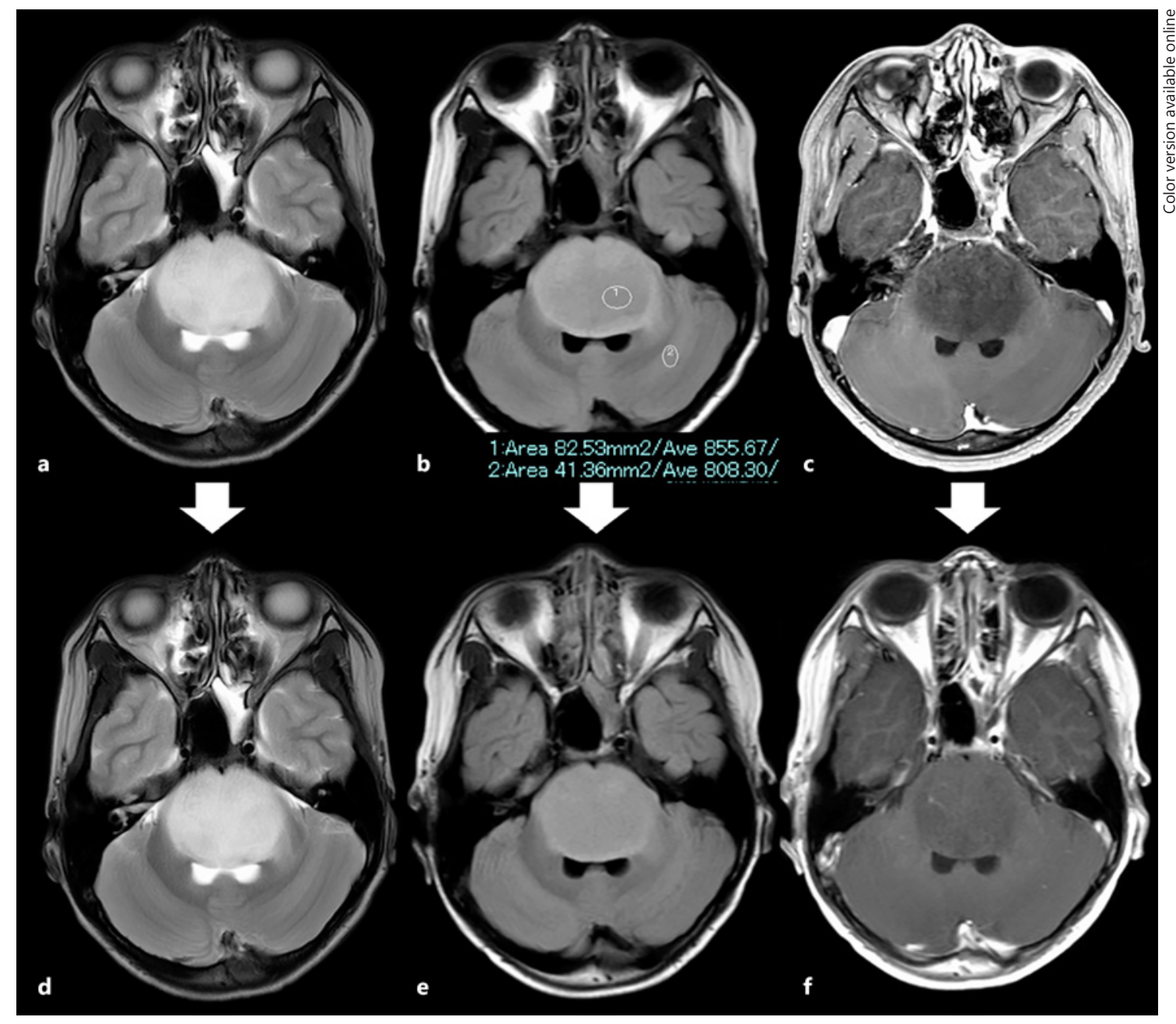

Fig. 5. Representative case of 6-year-old boy without T2-FLAIR mismatch sign in DIPG (case No. 13). Pre- $(\mathbf{a}-\mathbf{c})$ and post-RT (df) images. Axial T2WI (a, d), FLAIR images $(\mathbf{b}, \mathbf{e})$, and gadolinium-enhanced image $(\mathbf{c}, \mathbf{f})$ of a patient with DIPG. a T2WI of preradiotherapy demonstrates homogeneous high-intensity tumor throughout the lesion. b FLAIR image of pre-radiotherapy demonstrates iso- to high intensity at tumor and the signal intensity is higher compared to cerebellar cortex. The ratio of visually lower intensity inside the tumor and visually higher intensity at cerebellar cortex was 1.06 and confirmed that FLAIR intensity of tumor is higher than cerebellar cortex. c Gadolinium enhanced T1WI demonstrates homogenous low-intensity tumor with no enhancement. d-f Tumor showed no response after irradiation. DIPG, diffuse intrinsic pontine glioma; FLAIR, fluid-attenuated inversion recovery. urges defining the real patients' subgroup that can benefit from increased irradiation dose.

Recent development in molecular analysis revealed that histone H3 (H3F3A and HIST1H3B/C) K27M mutations are frequent in DIPGs, which arise in the brainstem almost exclusively in children, and in pediatric highgrade gliomas of midline structures such as thalamus and cerebellum, while G34R/V histone $\mathrm{H} 3$ mutations occur in pediatric high-grade gliomas of the cerebral cortex [22, 23]. Another analysis showed that patients with H3.1 (HIST1H3B/C) K27M mutation were associated with a better response to radiotherapy and had better OS compared to patients with H3.3 (H3F3A) K27M mutation [24]. These results are consistent with the reports that younger age at onset had a better outcome for children with diffuse pontine glioma [25]. These reports may be associated with our results that T2-FLAIR mismatch in DIPG developed at younger age than T2-FLAIR match.

T2-FLAIR mismatch sign has been described as good imaging biomarker for predicting diffuse astrocytoma, IDH-mutant in 2017 [6-10]. However, recent reports showed that this sign is not specific for diffuse astrocy- 
toma, IDH-mutant, but observed in many other gliomas including pilomyxoid astrocytoma, oligodendroglioma with IDH mutant and $1 \mathrm{p} / 19 \mathrm{q}$ codeleted, and low-grade astrocytoma harboring MYB rearrangement [11]. Diffuse midline glioma, H3 K27M-mutant at thalamus, was reported as one of the exceptions for this sign. Diffuse astrocytoma is of 2 subtypes: IDH-mutant subtype corresponding to T2-FLAIR mismatch and IDH-wild-type subtype corresponding to T2-FLAIR match. The T2FLAIR mismatch tumor, IDH-mutant diffuse astrocytoma, obviously has good prognosis compared to T2-FLAIR match tumors. These facts may be consistent with our results in DIPG.

Our study has some limitations. First, we did not confirm the histological subtype in our patient series. Surgical intervention for DIPG should be avoided as much as possible in nonclinical trial setting and even in clinical trial setting because the prognosis of DIPG is extremely poor. Second, we did not consider the effect of chemotherapy. Third, our patient population is too small for definitive conclusion. Future larger prospective studies are necessary to confirm the clinical value of T2-FLAIR mismatch in DIPG.

\section{Conclusion}

T2-FLAIR mismatch sign in DIPG may be an indicator for better response to radiotherapy, and these patients may be a potential candidate to be treated with increased effective biological dose of radiotherapy.

\section{Statement of Ethics}

This retrospective study was approved by our institutional review board approved this retrospective study (IRB No. 2922/E1650). Patients' written informed consent was waived.

\section{Conflict of Interest Statement}

The authors have no conflicts of interest to disclose.

\section{Funding Sources}

The authors did not receive any funding.

\section{Author Contributions}

Fumiyuki Yamasaki: the conception and design of the study and writing-original draft preparation. Ikuno Nishibuchi: investigation, writing-reviewing, and editing. Shuhei Karakawa: data curation. Yoko Kaichi: data curation, writing-reviewing, and editing. Manish Kolakshyapati: writing-reviewing and editing. Motoki Takano: data curation. Ushio Yonezawa: data curation. Nobuki Imano: data curation, writing-reviewing, and editing. Akira Taguchi: data curation. Maiko Shimomura: data curation. Maki Taniguchi: data curation. Shumpei Onishi: conceptualization and methodology. Satoshi Okada: supervision, writing-reviewing, and editing. Kaoru Kurisu: supervision. Kazuo Awai: supervision, writing-reviewing, and editing. Kazuhiko Sugiyama: supervision, writing-reviewing, and editing. Yasushi Nagata: supervision, writing-reviewing, and editing.

\section{References}

1 Rechberger JS, Lu VM, Zhang L, Power EA, Daniels DJ. Clinical trials for diffuse intrinsic pontine glioma: the current state of affairs. Childs Nerv Syst. 2020;36(1):39-46.

2 Pollack IF, Agnihotri S, Broniscer A. Childhood brain tumors: current management, biological insights, and future directions. J Neurosurg Pediatr. 2019 Mar 1;23(3):261-73.

3 El-Khouly FE, Veldhuijzen van Zanten SEM, Santa-Maria Lopez V, Hendrikse NH, Kaspers GJL, Loizos G, et al. Diagnostics and treatment of diffuse intrinsic pontine glioma: where do we stand? J Neurooncol. 2019 Oct; 145(1):177-84

4 Rashed WM, Maher E, Adel M, Saber O, Zaghloul MS. Pediatric diffuse intrinsic pontine glioma: where do we stand? Cancer Metastasis Rev. 2019 Dec 4;38(4):759-70.
5 Hoffman LM, Veldhuijzen van Zanten SEM, Colditz N, Baugh J, Chaney B, Hoffmann M, et al. Clinical, radiologic, pathologic, and molecular characteristics of long-term survivors of Diffuse Intrinsic Pontine Glioma (DIPG): a collaborative report from the International and European Society for Pediatric Oncology DIPG Registries. J Clin Oncol. 2018 Jul 1; 36(19):1963-72.

6 Patel SH, Poisson LM, Brat DJ, Zhou Y, Cooper L, Snuderl M, et al. T2-FLAIR mismatch, an imaging biomarker for IDH and $1 \mathrm{p} / 19 \mathrm{q}$ status in lower-grade gliomas: a TCGA/TCIA project. Clin Cancer Res. 2017 Oct 15;23(20): 6078-85.

7 Broen MPG, Smits M, Wijnenga MMJ, Dubbink HJ, Anten MHME, Schijns OEMG, et al. The T2-FLAIR mismatch sign as an imaging marker for non-enhancing IDH-mutant, 1p/19q-intact lower-grade glioma: a validation study. Neuro Oncol. 2018 Sep 3;20(10): 1393-9.
8 Lasocki A, Gaillard F, Gorelik A, Gonzales M. MRI features can predict $1 \mathrm{p} / 19 \mathrm{q}$ status in intracranial gliomas. AJNR Am J Neuroradiol. 2018 Apr;39(4):687-92.

9 Batchala PP, Muttikkal TJE, Donahue JH, Patrie JT, Schiff D, Fadul CE, et al. Neuroimaging-based classification algorithm for predicting $1 \mathrm{p} / 19 \mathrm{q}$-codeletion status in IDH-mutant lower grade gliomas. AJNR Am J Neuroradiol. 2019 Mar;40(3):426-32.

10 Juratli TA, Tummala SS, Riedl A, Daubner D, Hennig S, Penson T, et al. Radiographic assessment of contrast enhancement and T2/ FLAIR mismatch sign in lower grade gliomas: correlation with molecular groups. $\mathrm{J} \mathrm{Neu}-$ rooncol. 2019 Jan;141(2):327-35.

11 Johnson DR, Kaufmann TJ, Patel SH, Chi AS, Snuderl M, Jain R. There is an exception to every rule-T2-FLAIR mismatch sign in gliomas. Neuroradiology. 2019 Feb;61(2):225-7. 
12 Yamasaki F, Kurisu K, Kajiwara Y, Watanabe Y, Takayasu T, Akiyama Y, et al. Magnetic resonance spectroscopic detection of lactate is predictive of a poor prognosis in patients with diffuse intrinsic pontine glioma. Neuro Oncol. 2011 Jul;13(7):791-801.

13 Kuzan-Fischer CM, Souweidane MM. The intersect of neurosurgery with diffuse intrinsic pontine glioma. J Neurosurg Pediatr. 2019 Dec 1;24(6):611-21.

14 Warren KE. Diffuse intrinsic pontine glioma: poised for progress. Front Oncol. 2012;2:205.

15 Lassaletta A, Strother D, Laperriere N, Hukin J, Vanan MI, Goddard K, et al. Reirradiation in patients with diffuse intrinsic pontine gliomas: the Canadian experience. Pediatr Blood Cancer. 2018 Jun;65(6):e26988.

16 Fontanilla HP, Pinnix CC, Ketonen LM, Woo SY, Vats TS, Rytting ME, et al. Palliative reirradiation for progressive diffuse intrinsic pontine glioma. Am J Clin Oncol. 2012 Feb; 35(1):51-7.
17 Khatua S, Zaky W. Diffuse intrinsic pontine glioma: time for therapeutic optimism. CNS Oncol. 2014;3(5):337-48.

18 Massimino M, Biassoni V, Miceli R, Schiavello E, Warmuth-Metz M, Modena P, et al. Results of nimotuzumab and vinorelbine, radiation and re-irradiation for diffuse pontine glioma in childhood. J Neurooncol. 2014 Jun; 118(2):305-12.

19 Janssens GO, Gandola L, Bolle S, Mandeville H, Ramos-Albiac M, van Beek K, et al. Survival benefit for patients with diffuse intrinsic pontine glioma (DIPG) undergoing re-irradiation at first progression: a matched-cohort analysis on behalf of the SIOP-E-HGG/DIPG working group. Eur J Cancer. 2017 Mar;73: 38-47.

20 Amsbaugh MJ, Mahajan A, Thall PF, McAleer MF, Paulino AC, Grosshans D, et al. A phase $1 / 2$ trial of reirradiation for diffuse intrinsic pontine glioma. Int J Radiat Oncol Biol Phys. 2019 May $1 ; 104(1): 144-8$

21 Lu VM, Welby JP, Mahajan A, Laack NN Daniels DJ. Reirradiation for diffuse intrinsic pontine glioma: a systematic review and meta-analysis. Childs Nerv Syst. 2019 May;35(5): $739-46$.
22 Schwartzentruber J, Korshunov A, Liu XY, Jones DT, Pfaff E, Jacob K, et al. Driver mutations in histone H3.3 and chromatin remodelling genes in paediatric glioblastoma. Nature. 2012 Jan 29;482(7384):226-31.

23 Wu G, Broniscer A, McEachron TA, Lu C, Paugh BS, Becksfort J, et al. Somatic histone $\mathrm{H} 3$ alterations in pediatric diffuse intrinsic pontine gliomas and non-brainstem glioblastomas. Nat Genet. 2012 Jan 29;44(3):251-3.

24 Castel D, Philippe C, Calmon R, Le Dret L, Truffaux N, Boddaert N, et al. Histone H3F3A and HIST1H3B K27M mutations define two subgroups of diffuse intrinsic pontine gliomas with different prognosis and phenotypes. Acta Neuropathol. 2015 Dec;130(6):815-27.

25 Broniscer A, Laningham FH, Sanders RP, Kun LE, Ellison DW, Gajjar A. Young age may predict a better outcome for children with diffuse pontine glioma. Cancer. 2008 Aug 1;113(3):566-72. 